\title{
تطبيق إدارة البرنامج المكثف العربي في جامعة تولونج أغونج الإسلامية الحكومية
}

\author{
أحمد نور خليص \\ مدرس جامعة تولونج أغونج الإسلامية الحكومية \\ Email : cholisahmad87@gmail.com
}

\begin{abstract}
ملخص
ينطلق هذا البحث من أن كثيرا من المؤسسات التربوية الإسلامية اليوم تعقد البرامج المكثفة الفعالة لترقية جودة التعليم واللغة العربية خاصة. وأهم هدف منها إعداد الطلاب القادرين على مواجهة المنافسة في المستقبل. لذلك، أدارت الجامعة الإسلامية الحكومية تولونج أغونج بوساطة مركز اللغة برنابحا مكثفا عربيا بالإستراتيجيات الخاصة، منها بتفوق الرسالة، والاستراتيجية لبلوغ الرسالة، ومشاركة جميع الزبائن داخلية كانت أم خارجية في تنمية الاستراتيجية، وتقوية الموظفين بتحريك الميول إلى إصدار المساهمة

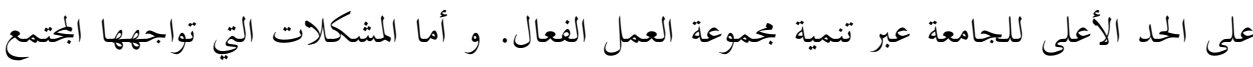
الأكادمي هي أن المقياس المهني الذي يكون مطلوبا في جميع المهن غير محققة عند ما تخرج الطلبة في الجامعة، وبعبارة أخرى أها تجب أن تكون هناك محاولة على ترقية جودة اللغة العبية. وأما طريقة البحث هو بحث كيفي ميداني (Grounded). وأساليب جمع البيانات هي الملاحظة والمقابلة والوثائق. والخلاصة هي أن إجراء البرنامج المكثف العربي في الجحامعة الإسلامية الحكومية تولونج لم يزل غير جيد، ذلك لما كانت المشكلات تبدو في أشياء وهي عدم التنسيق بين العناصر في تلك الجامعة وعدم الاستمرار في سلسلة المواد الدراسية والاستعمال اللغوي في التدريس.
\end{abstract}

\section{ABSTRACT}

This study contradicts the fact that nowadays many educational institutions conducting an intensive program that is effective for improving the quality of learning Arabic. The goal is to prepare students to have the ability to compete in the face of competition in the future. Therefore, STAIN Tulung manage intensive Arabic program with a clear vision and mission, strategically where, the participation of all elements of the school to make the maximum contribution to the strengthening of institutions. The problem is a graduate of the institution have not reached a satisfactory quality, are still required to efforts to improve the quality of learning Arabic. The method used in this research is grounded 
research. Data collection techniques used were observation, interviews and documentation. The results showed that penyenggaraan intensive Arabic program at STAIN Tulung remains effective for several reasons, in ataranya there is no compatibility between the various elements in the institutions and the lack of continuity between the material being taught with the use of language in learning.

Keywords: implementation, management, intensive language program, professional

أصبحت دراسة اللغة العربية المكثفة عنصرا هامّا في فهم العلوم والفنون مثل التفسير والحديث

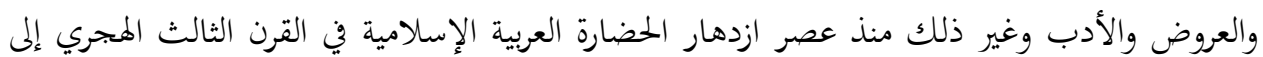

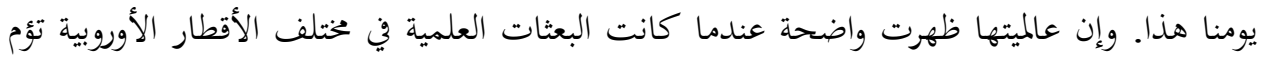

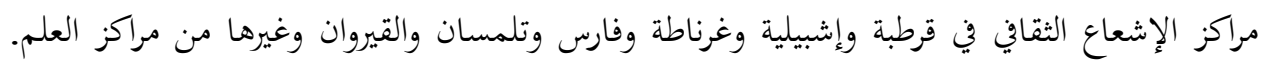

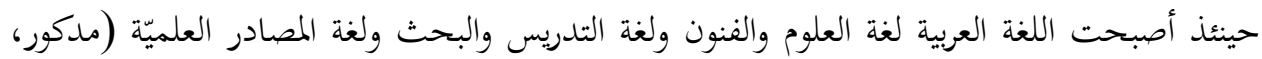

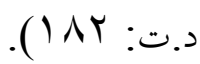
تعدّ دراسة اللغة العربية المكثفة في بلاد أندونيسيا من أهم مقومات الثقافة الإسلامية. وهم

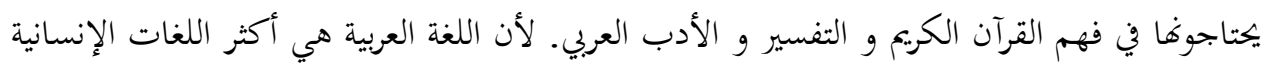

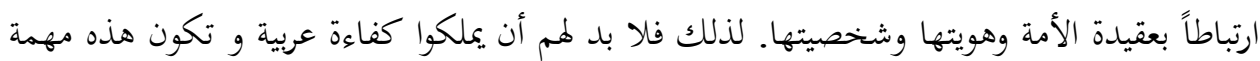
في الاتصال اليومي مع الناطقين بها الذين حضروا من الدول العربية. و يستطيع المسلمون الإندونيسيون

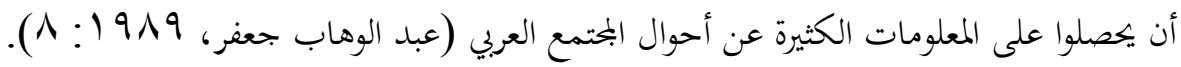

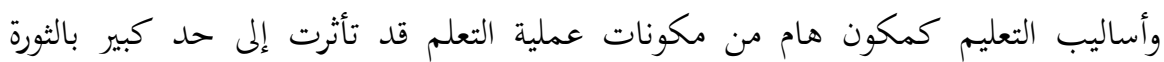

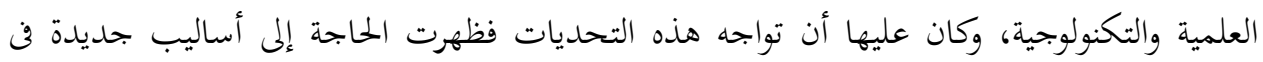

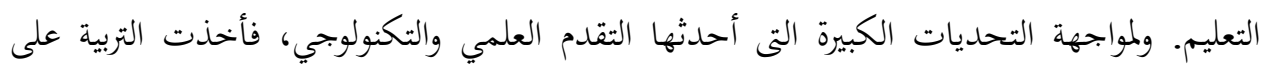

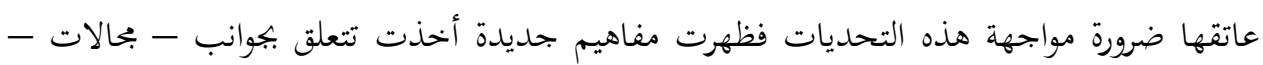

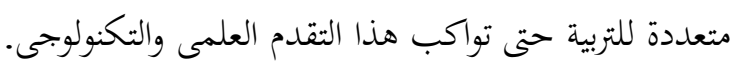
وتعد أساليب التدريس أحد البحالات المامة للتربية التى تأثرت بتلك التكات الاتحاهات الحديثة سواء

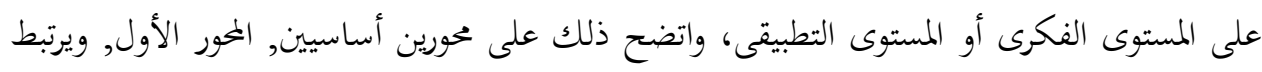
بالجانب الفكرى, والمحور الثان يرتبط بالجانب التطبيقى في المواقف التعليمية.

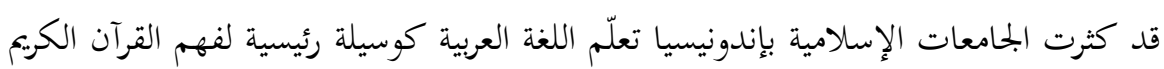


والحديث النبوي والكتب الإسلامية. ولكن الواقع، لا يزال التعليم يرشب لجعل الطلاب القادرين على فهم اللغة العربية والتكلم بها فعالا. من إحدى العوامل هي طول زمان الدراسة والملل الذي يعوق رغبة الطلاب في تعلم اللغة العربية. ذلك لما كانت الحصة الدراسية لم تزد من حصتين إلى ثلاث حصص في الأسبوع، من هذا الجحانب قد ظهر الفشل في إجراء برنامج اللغة العربية الفعال.

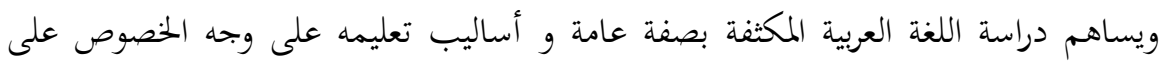
تطوير إمكانات الطلبة بما يمكنه من مواجهة هذه التحديات الهائلة والتعامل معها بفكر منظومى شامل وليس بفكر أحادى أو ثنائى التوجه، وهو ما يستلزم إعداد أجيال المستقبل بذلك الفكر. وتزيد تلك المشكلة بكفاءة معظم المدرسين من إندونيسا، حيث كانوا ماهرين في قواعد اللغة العربية نظرية، ولكن من جهة الذوق اللغوي أن كفاءقم محدودة جدا. كثير من مدرسي اللغة العربية يستطيعون أن يتكلموا باللغة العربية، ولكن بدون التعبير العربي الذي ينطق به العرب. حتى يكون

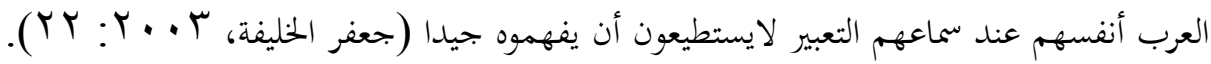
والمنهج الدراسي الذي لايتم اندماجه جيدا يكون عاملا مانعا كبيرا لنجاح المكثف العربي. وهو عندما وجب على الطلاب أن يتعلموا أنواع الأنظمة والرموز والقواعد وغيرها، وكان تطبيقها

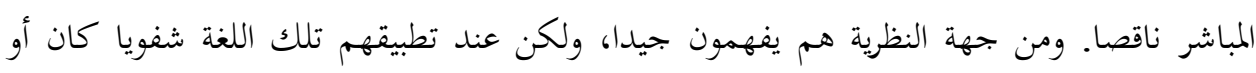
تحريريا أو استماعيا، كانوا يبتعدون عن الأهلية المرجوة.

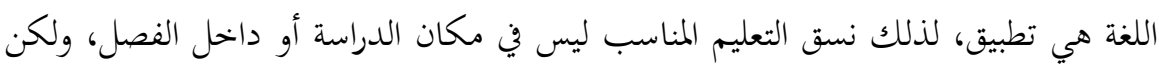
عن وسيلة التطبيق المباشر جماعيا أو بوجود البيئة اللغوية حيث يتحاور جميع المتعلمين بتلك اللغة، حتى يقال أن هذا أهم من تعلم التكلم بتلك اللغة.

وباختصار القول أن تعلم اللغة يحتاج إلى بيئة يتكلم فيها متعلموا تلك اللغة. حيث نكون فيها

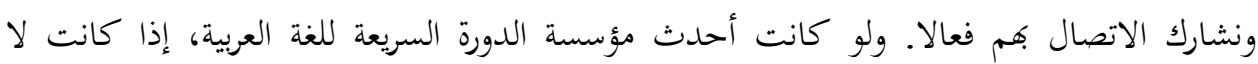
تستطيع تكوين البيئة العربية، فطبعا لا تستطيع توليد المتخرج الماهر في اللغة العربية. لذلك، أدارت الجامعة الإسلامية الحكومية تولونج أغونج بوساطة مركز اللغة برنابحا مكثفا عربيا باستراتيجية الجودة، منها بتفوق الرسالة، وتركيز الزبون واضحا، والاستراتيجية لبلوغ الرسالة، ومشاركة جميع الزبائن داخلية كانت أم خارجية في تنمية الاستراتيجية، وتقوية الموظفين بتحريك الميول إلى إصدار المساهمة على الحد الأعلى للجامعة عبر تنمية بحموعة العمل الفعال، وكذلك تقييم وتقويم فعالية الجامعة في مواجهة الهدف الذي يرجوه الزبون. 
الطريقة المستخدمة في هذا البحث هو بحث كيفي ميداني (Grounded Reshearch)

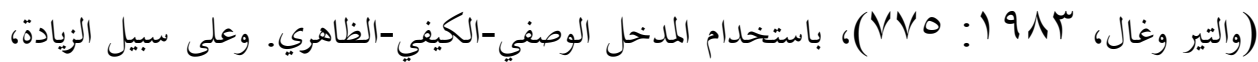

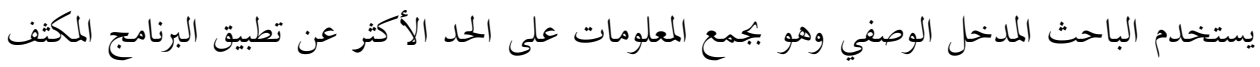

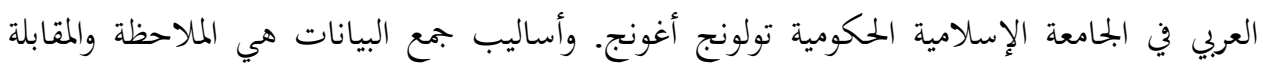
والوثائق.

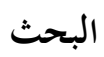

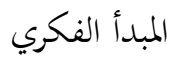

تطبيق إدارة البرنامج المكثف العربي في الجامعة الإسلامية الحكومية تولونج أغونج على الأساس

$$
\text { التالي: }
$$

نظرية التعلم السلوكي هي عملية تغيير السلوك سببا لوجود الاتصال بين المثير والإجابة الذي يسبب الطلاب على ملك الخبرة الجديدة. وتطبيقها في التدريس على أن المدرس لديه كفاءة في إدارة

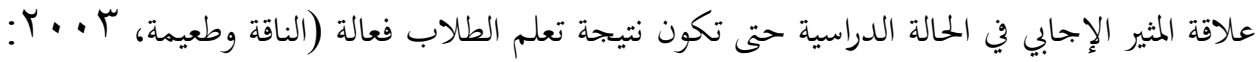

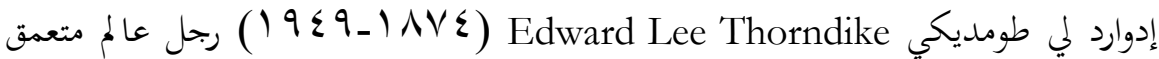
في نطرية التعلم السلوكي. هو مدرس وعالم في علم النفس وأمريكي الجنسية قال أن التعلم عملية

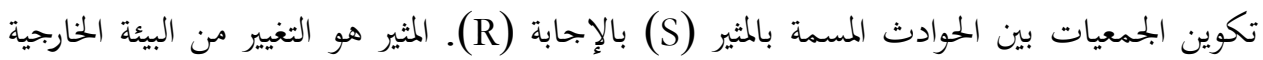

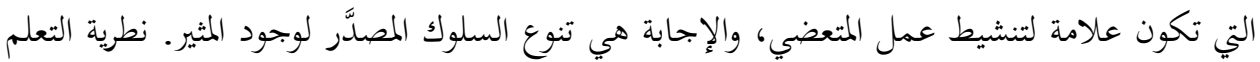

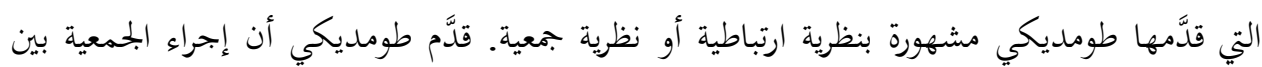

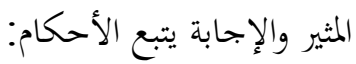
أ. الحكم الاستعدادي (Law of Readiness)، إذا كان المتعضي أكثر استعدادا لنيل تغيير السلوك فإجراء السلوك سيصدّر الاقتناع النفسي حتى تكون الجمعية تميل إلى تأكيدها.

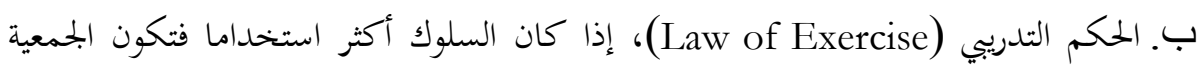
أشد قوة. ج. الحكم العقبي (Law of Effect)، علاقة المثير الاجابي تميل إلى تأكيده إذا كانت عاقبتها مفرَّحة ومائلة إلى تضعيفها إذا كانت عاقبتها غير مقنعة. 
r

المبدأ الديني عن تطبيق إدارة البرنامج المكثف العربي.

سورة يوسف آية 1 • 1 : "قل هذه سبيلي أدعو إلى الله على بصيرة أنا ومن اتبعني وسبحان

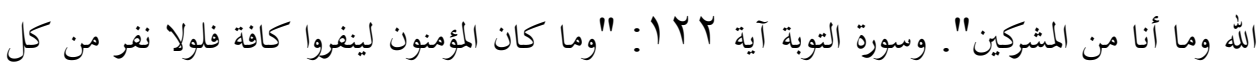

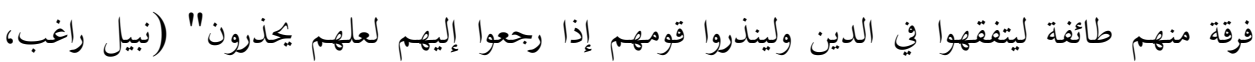

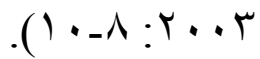

المبدأ من الحديث النبوي الشريف عن تطبيق إدارة البرنامج المكثف العربي برواية أبي هريرة رضي الله عنه: "تعلموا اللغة العربية وعلموها الناس" (رواه أبو هريرة) ب. أمبدأ القضائي

أما المبدأ القضائي عن تطبيق البرنامج المكثف العربي هو وجود الإيضاح المتعلق بتدريس اللغة العربية في الجامعة الإسلامية كما وجدنا في ملحق تقرير مدير الجنرال لإشراف المؤسسة الإسلامية رقم

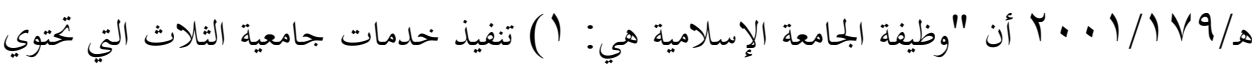

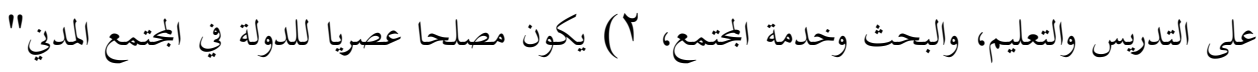

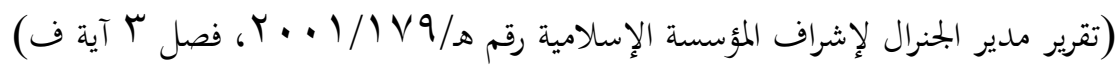
وبالتفصيل للمبدأ القضائي عن تطبيق البرنامج المكثف العربي بجامعة تولونج أغونج الإسلامية الحكومية فيما يلي:

Nomor SK pendirian

Tanggal SK pendirian

Pejabat Penandatangan

SK Pendirian

Nomor SK Izin Operasional : Dj.I/1457/2002

Tanggal SK Izin Operasional : 25 Oktober 2002

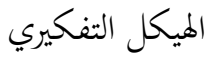

\begin{tabular}{|c|c|c|}
\hline & علوم اللغة الحديثة & \\
\hline علم اللغة التطبيقي & & $\begin{array}{c}\text { علم اللغة النظري العام } \\
\text { (Theoretical Linguistics) }\end{array}$ \\
\hline
\end{tabular}


Ahmad Nurcholis

صدر تطبيق إدارة البرنامج المكثف العربي من التفكير أن علم اللغة التطبيقي يمكن وجود

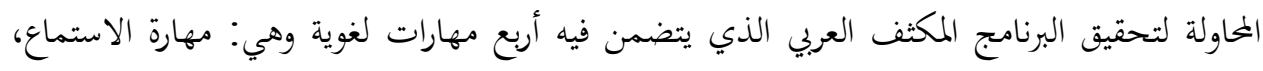

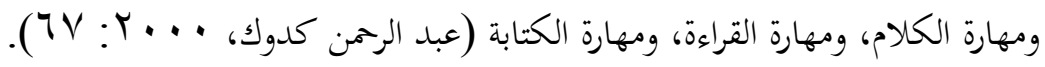

$$
\text { نظرية إدارة البرنامج المكثف العربي }
$$

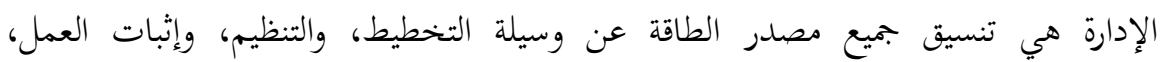
والتوجيه، والمراقبة للبلوغ إلى المدف المقرر أولا. وظيفة الإدارة هي مبادئ أساسية موجودة دائما

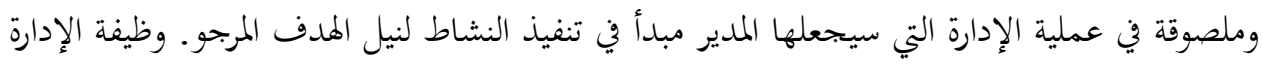

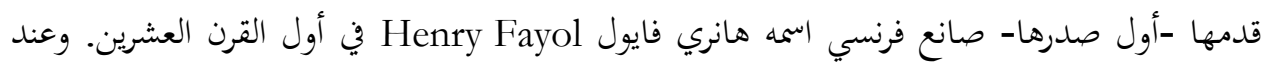

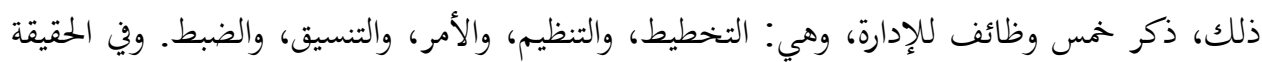

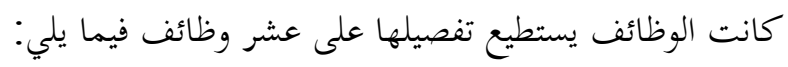
l. التنبؤ، وهو نشاط تنبئي، والتصوير بالتمكن عند إجرائه.

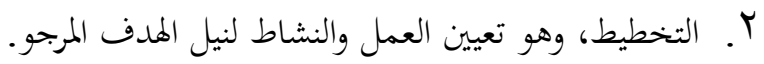

؟ّ. التنظيم، وهو جمع النشاط لنيل المدف، وفيه تعيين هيكل النتظيم، الواجبة ووظيفتها.

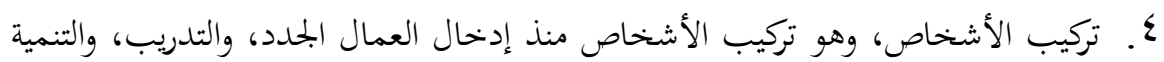
حتى المحاولة ليكون العمال لديهم كفاءة عالية فعالة في الجمعية.

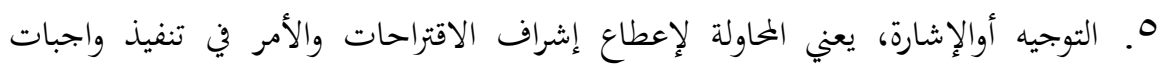
العمال للقيام بها جيدا وصحيحا مناسبا بالهدف المقرر.

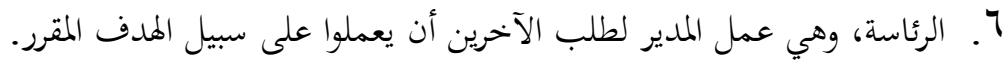

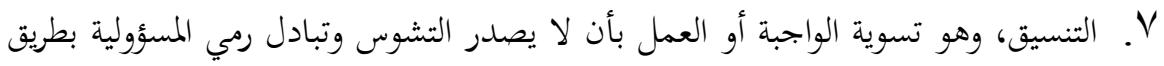
إيصال، وتوحيد، وتسوية واجبات العمال. ^. التشجيع، وهو إعطاء الحماسة، والإلهام، والدفع إلى العمال بأن يعملوال الواجبات المقرر مخلصين. 9 9. المراقبة، وهي إيجاد وتطبيق الطريقة والآلة للتأمين على أن التخطيط قد عقد مناسبا بالهدف. • ( التبليغ، وهو إلقاء نتيجة النشاطات تحريريا كان أو شفويا (البشاري، ... ب. . 
إجراء البرنامج باستخدام الإدارة المتفوقة ستؤدي إلى النتيجة المتفوقة أيضا، لذلك كثير من المؤسسات لاتسير فعالة بل فاشلة لأن الوظيفة السابقة غير مراقبة جيدا أو كانت مراقبتها ضعيفة تسبب كذلك إلى نتيجة ضعيفة.

لتأكيد الخطوة في تدبير المؤسسة الإسلامية، أرشد هـأ.ر تيلائار H.A.R Tilaar إلى أن تدبير التربية الإسلامية ينبغي لها الاهتمام بأربع خطوات مفضلة فيما يلي: الأولى، ترقية الجودة. والثانية،

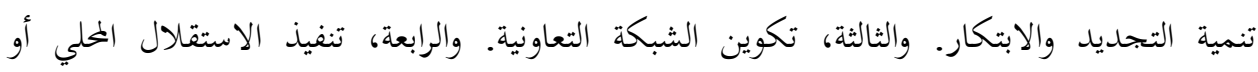

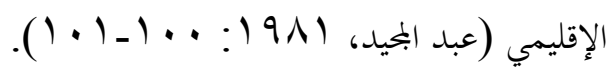

للحصول على نتيجة البرنامج المكثف العربي بالإدارة الجيدة مثل السابق، فالحاجة إلى تكوين

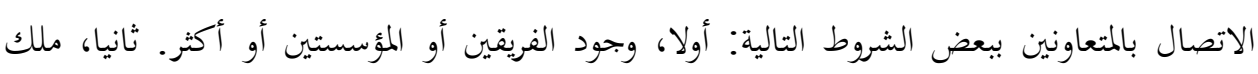

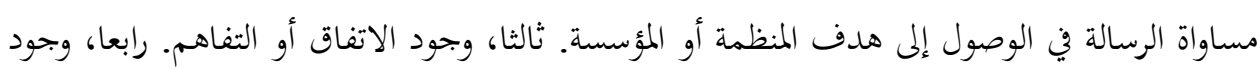
تبادل الاعتقاد والاحتياج. خامسا، وجود التعهد الجماعي للوصول إلى الهدف الأكبر. هناك أربعة عمدان في تنظيم مؤسسة وتكون مبدأ في إجراء عمليتها، وهي: توزيع العمل، وتصنيف العمل، وتعيين العميل بين الأقسام، والتنسيق.

لإنتاج البرنامج المكثف العربي الأفضل، فالحاجة إلى الإدارة الجيدة ولديه مبدأ فلسفي صحيح حسب تأسيس تلك المؤسسة. إدارة برنامج التدريس باستخدام المدخل النظامي مبدأ فلسفيا وبتريبيا ينبغي لها وجود العناصر التالية:

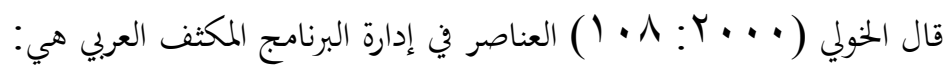

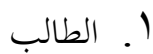

عند إجراء البرنامج المكثف العربي، ينبغي أن تكون فيه إعلانات وقوة التنافس العالي للحصول على الطالب المرجو واستيفاء المعيار المطلوب.

$$
\text { ץ ب . خطة البرنامج المكثف العربي }
$$

ينبغي للمنهج الدراسي المخطَّط مناسب بتقويم الحاجة المعمول سابقا، وهو إذا كان هدف لئف البرنامج لتسهيل البحتمع في العمل خارج البادد فتكوين المنهج الدراسي على أساس العمل، وإذا كان الهدف لإعدادهم متعمقين في المهارات اللغوية فتكوين المنهج الدراسي على أساس سداد وتوافق المادة. ب إم المدرس 
Ahmad Nurcholis

آجر المدرس الذي يكون عنده أهلية تربوية ومؤهل بها. على الأقل كان المدرس متخرجا وماجستيرا

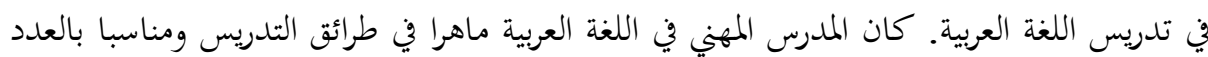
والمعيار المتناج إليه. ع. ع. الوسيلة

عند تأسيس البرنامج المكثف ينبغي أن يكون هناك مكان ووسيلة ووسائل تدريس اللغة العربية المناسب لكي يشعر الطالب بالاقتناع ويساعد على نجاح المدرسين لإعطاء النتيجة الجيدة لمتمخرجين فيه.

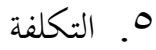

لتحقيق تأسيس البرنامج المكثف، ينبغي أن تكون هناك تكلفة كافية لكي يجري البرنامج على ما

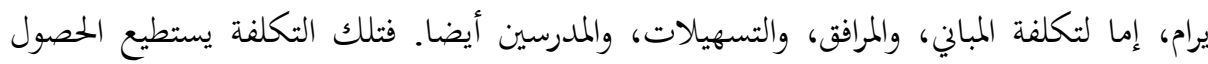

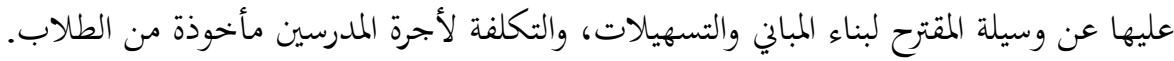
T. الثقافة

عادة لكل البرنامج المكثف علامة ثقافة خاصة تساعد على نجاح عملية التدريس، لذلك يجب

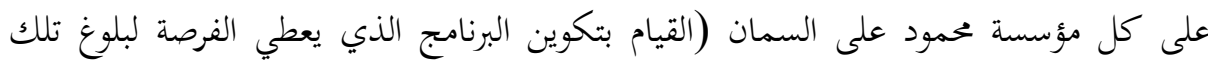

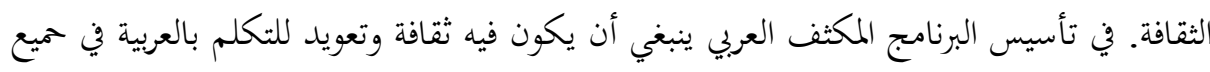

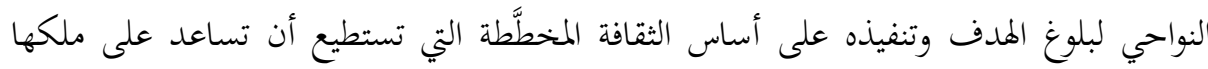
وتنميتها. V

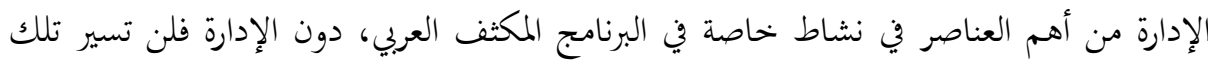

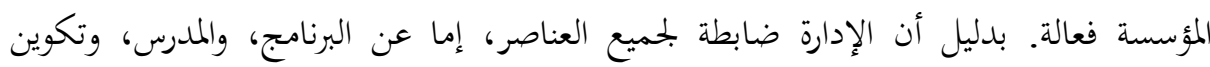
الثقافة، وتنظيم الإدارة وغيرها.

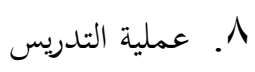

لا تتساوى عملية تدريس العربية في البرنامج المكثف العربي بتدريس المادة العادية، لأن في البرنامج

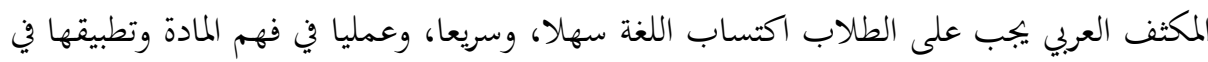

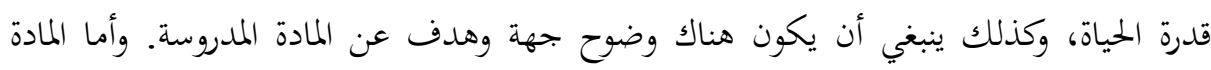

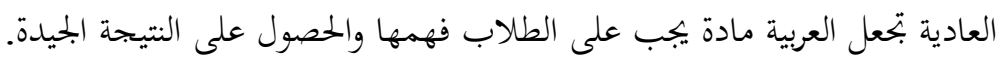




$$
9
$$

بإجراء جميع الأنظمة السابقة، فالمرجو منه أن يحصل الطلاب على النتيجة الممتازة حتى يشعروا

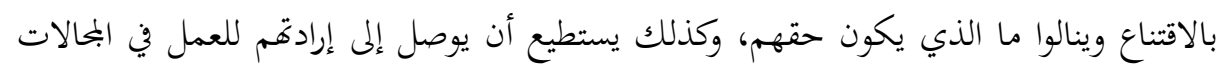

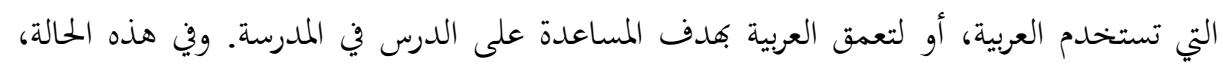
كانت الحاجة إلى التعليق بالتقويم.

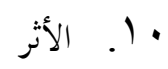

بعد انتهاء البرنامج المكثف، فالمطلوب من الطلاب أن يحصلوا على الأثر النافع عاقبة للمؤسسة

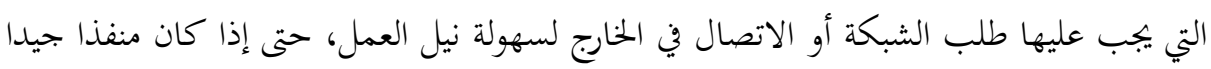

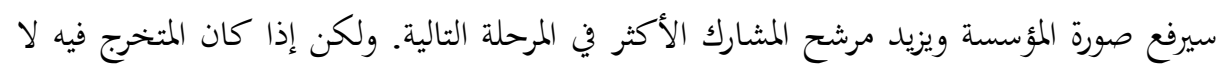

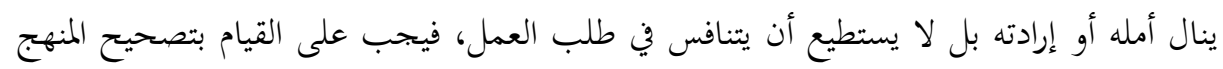

$$
\text { الدراسي لبلوغ الهدف المرجو. }
$$

إذا تحدثنا عن البيئة فطبعا تحدثنا كذلك عن زمان المستقبل والأنشطة بعد التخرج، وتحتاج البيئة

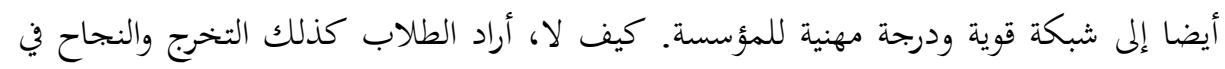

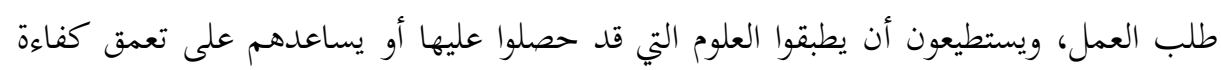

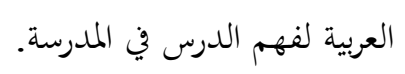

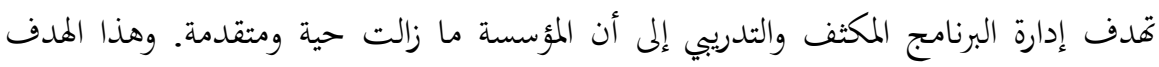

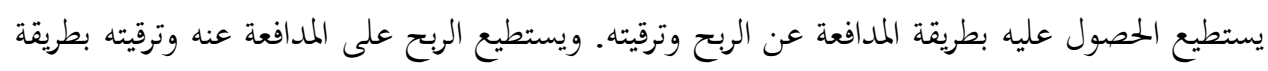

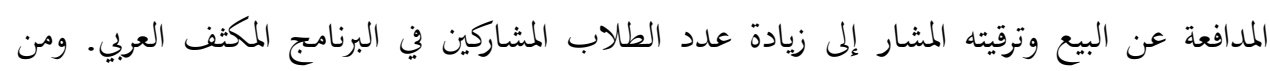
إحدى الطرائق للمدافعة عن البيع وترقيته بترقية النشاط التسويقي تكثيفا واستمرارا.

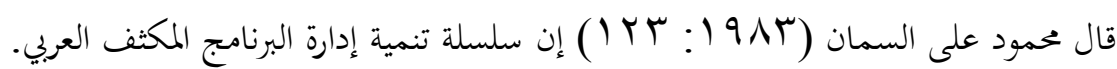

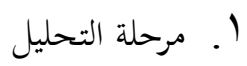

أ. التعرُف وتحليل حاجة (Need Assessment) البرنامج المكثف العربي وكذلك تعرُف النتيجة

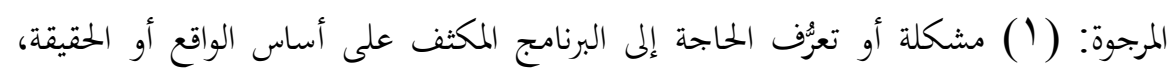

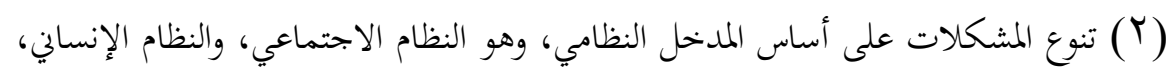
ونظام المؤسسة، والنظام الإعلامي، والنظام التكنولوجي. 
Ahmad Nurcholis

ب. تحليل الواجبة، مثلا واجبة ودور الشخص أو جماعة من الناس، وتعرُّف النتيجة المرجوة: (1)

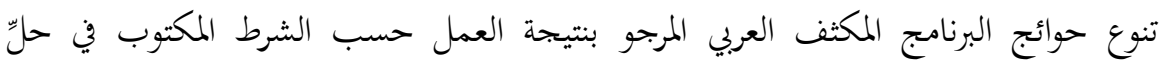

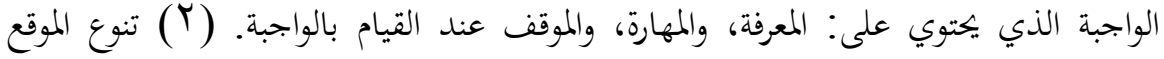

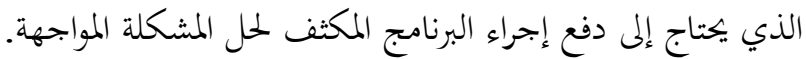

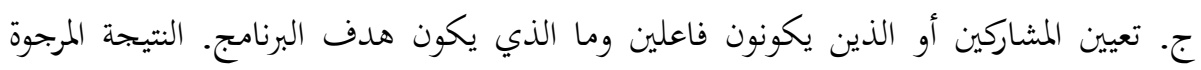

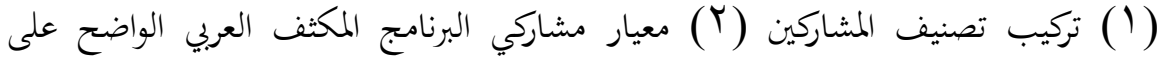

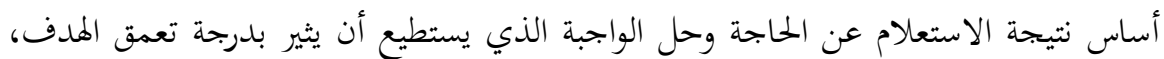
وتركيب المادة واختار طريقة التدريس. r ب مرحلة التنمية

التنمية في المقترح الذي يكتوي على ( (1) الحلفية أو المقدمة التي تبين أنواع المشكلات المواجهة.

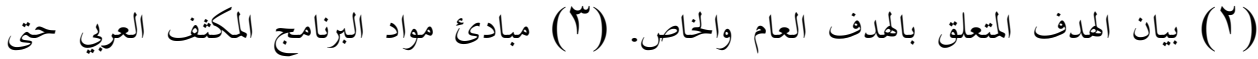

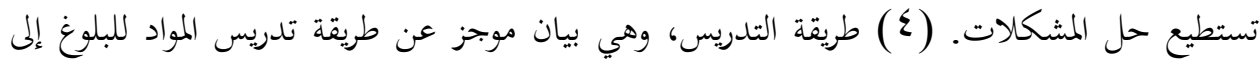

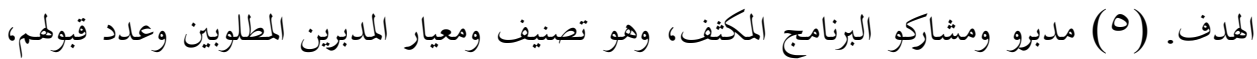

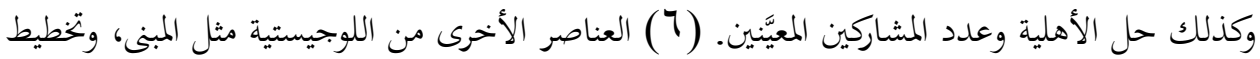

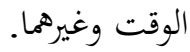

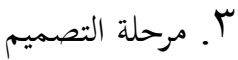
أما إجراء التصميم فيما يلي: (1) (1) رمز الهدف. (Y) تخطيط البرنامج (عن المضمون،

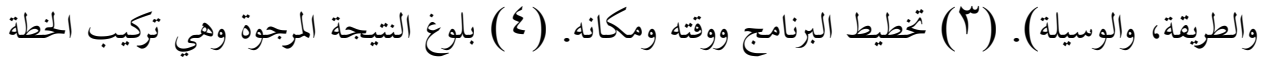

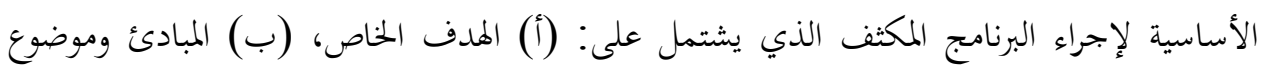
البحث، (ج) الطريقة والوسيلة أو تسهيلات البرنامج المكثف، (د) معيار وخطة قبول المشاركين، (هـ)

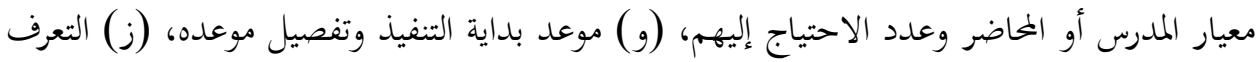

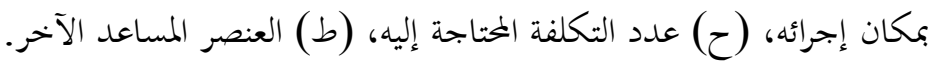
ع. مرحلة التطبيق

وهي إجراء البرنامج بما كتب في خطة البرنامج. وفي هذه المرحلة تحتاج إلى سيناريو البرنامج

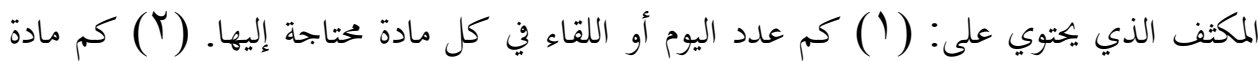
تقدم في كل لقاء. (r) تفصيل سيناريو أنشطة البرنامج المكثف أو دليل الإجراء والدليل الصناعي في ماده 


$$
\text { الذي سيقوم بتنفيذه. }
$$

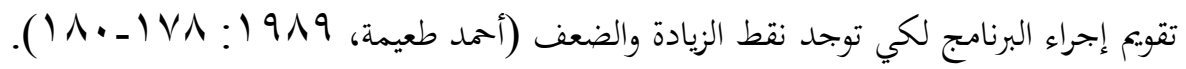

$$
\text { ץ ب. مرحلة الإعادة والتصحيح }
$$

عن وسيلة التقويم ستوجد الإعادة ومنها سيعقد تصحيح البرنامج للقيام بتنفيذه في المرحلة التالية.

$$
\text { تطبيق إدارة البرنامج المكتف العربي في الجامعة الإسلامية الحكومية تولونج أغونج }
$$

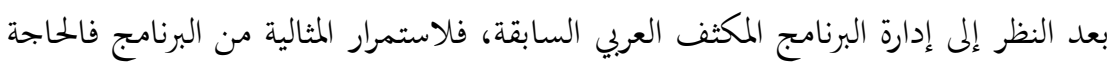

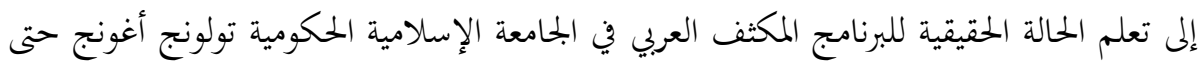

$$
\text { تكون مرجعا في إصلاح وبناء الإدارة الجيدة. }
$$

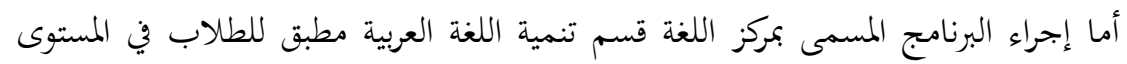

\begin{tabular}{|c|c|c|}
\hline 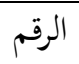 & نظرية وظيفة الإدارة & وظيفة الإدارة في مركز اللغة \\
\hline 1 & التنبؤ & 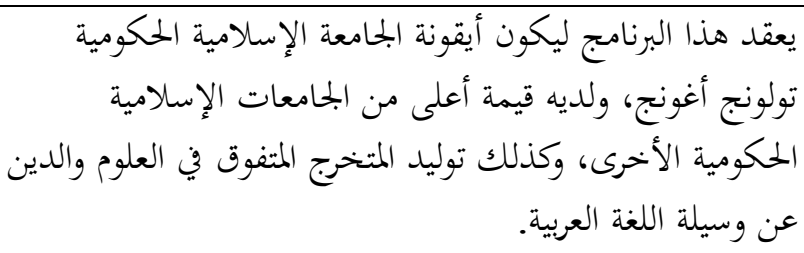 \\
\hline T & التخطيط & 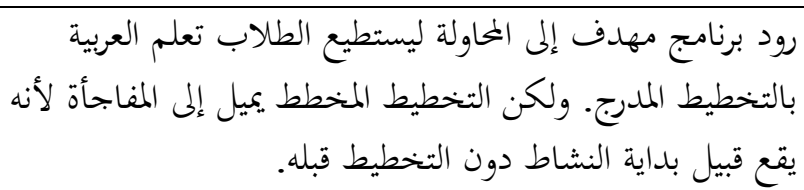 \\
\hline$\Gamma$ & التنظيم & 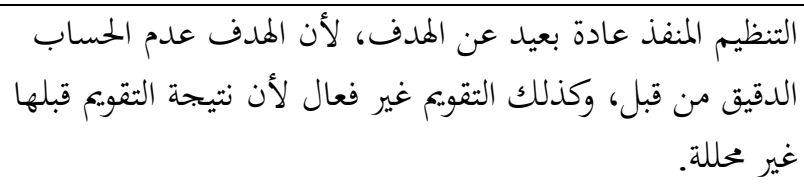 \\
\hline$\varepsilon$ & تركيب الشخصية & التدريس ليكون الانتقاء لمرشح المدرس بالاهدف يجمري على ما يرام. إلى فهم المادة وكفاءة \\
\hline
\end{tabular}

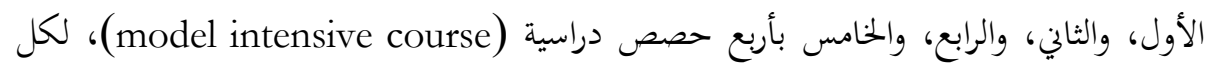

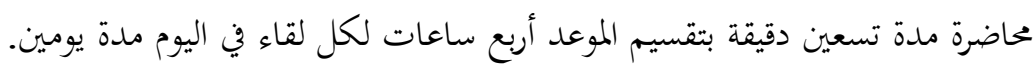

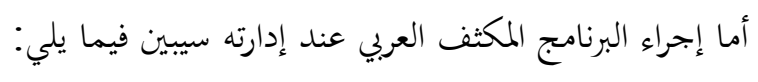

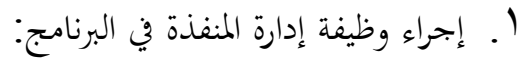


Ahmad Nurcholis

\begin{tabular}{|c|c|c|}
\hline 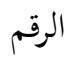 & نظرية وظيفة الإدارة & وظيفة الإدارة في مركز اللغة \\
\hline 0 & 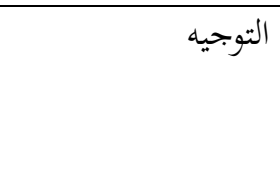 & ألتئيس. أما التوجيه غيرج الفعال، ينبغي أن يكون هناك توجيه من المدير أو \\
\hline 7 & الرئاسة & 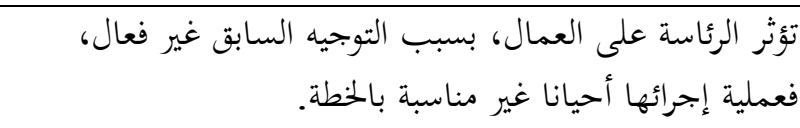 \\
\hline$V$ & التنسيق & إجراء التنسيق أحيانا متغير بمعنى نتيجة التنظيم يستطيع تغييرها، \\
\hline$\Lambda$ & التشجيع & و إعطاء الجائزة هم يستطيع كثير ألقاه المدير لترقية عمل شخصيته. بالثناء \\
\hline 9 & المراقبة & 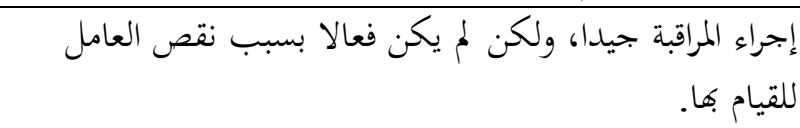 \\
\hline $1 \cdot$ & الإخبار & إجراء إخبار البرنامج إلى المدير عن نشاط البرنامج. \\
\hline
\end{tabular}

Y. إجراء الخطوة المفضلة لتأسيس البرنامج المكثف العربي:

\begin{tabular}{|c|c|c|}
\hline 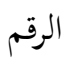 & الخطوة المفضلة & الخطوة المفضلة في مركز اللغة \\
\hline 1 & ترقية الجحودة & ترقية كفاءة اللغة العربية للطلاب المدرب الانتقاء بالاعتماد على شرط، وكذلك \\
\hline r & توالابتمار التجديد & القيام بعملية التدريس بطلب التجديد وإعطاء الفرصة الابتكارية \\
\hline r & بناء الشبكة التعاونية & بالكلية لكي يكون الاتصال بالمعهد بوصفه مكان تعويد العربية، وكذلك الإسلام باستخدام العربية. \\
\hline$\varepsilon$ & تنفيذ الاستقلال المحلي & مركز اللغة تعيين السياسة عن إجراء وتطبيق التدريس حق استقلالي \\
\hline
\end{tabular}

r. محاولة بناء الشبكة التعاونية

\begin{tabular}{|c|c|c|}
\hline الرقم & محاولة بناء الشبكة التعاونية & المحاولات في الجحامعة الإسلامية الحلكومية تولونج أغونج \\
\hline 1 & ترقية مشاركة المحتمع & تحاول الجامعة على الاتصال الجيد بابلمتمع وتدعوهم إلى لئل \\
\hline
\end{tabular}




\begin{tabular}{|c|c|c|}
\hline$r$ & ترقية الجودة والاتصال به & مرتفعة، وكاء الفرصة للمدرس ليلتحق دراسته لتكون إعطاء الجائزة عن وسيلة البحث لكودة تدريسه \\
\hline r & 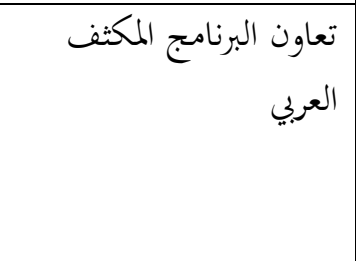 & 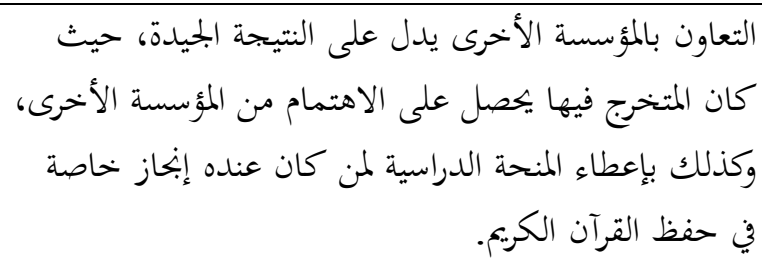 \\
\hline$\varepsilon$ & ترقية كفاءة المتخرج & إجراء التعاون بالمؤسسات الأخرى ليكون المتخرجون فيها \\
\hline 0 & الإعلان والترويج والنشر & 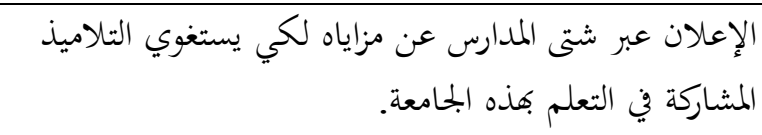 \\
\hline 7 & ترقية وسيلة الوصول & تنمية الشبكة وصلة الرحم لكي تصدر وسيلة الوصول الواسعة \\
\hline V & تصوير صورة للجمهور & المحاولة لحفظ السمى أو الصيت خارج الجامعة بعقد الاتصال \\
\hline$\Lambda$ & تقوية & تقوية قدرات الجحامعة بترقية وسائل وتسهيلات وقابلية الجامعة. \\
\hline
\end{tabular}

ع. ع عناصر إدارة البرنامج المكثف العربي

\begin{tabular}{|c|c|c|}
\hline الرقم & عناصر إدارة البرنامج المكثف & العناصر في مركز اللغة قسم اللغة العربية \\
\hline 1 & الطاباب & الملكثف. المشاركون جميع طلاب ألمستوى الأول، والثاني، والرابع، لمب موعد خحاص لتعلم العربية في البرنامج \\
\hline$r$ & البرنامج المكثف العربي & 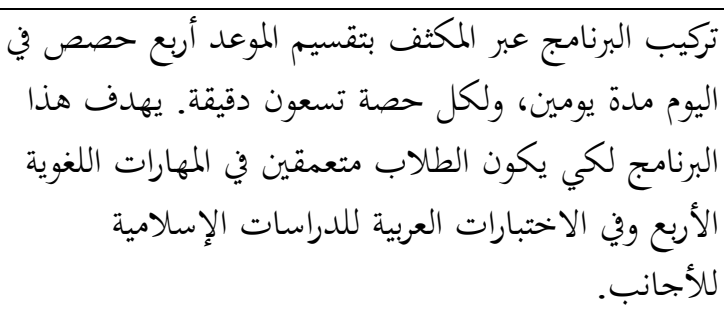 \\
\hline
\end{tabular}


Ahmad Nurcholis

\begin{tabular}{|c|c|c|}
\hline الرقم & عناصر إدارة البرنامج المكثف & العناصر في مركز اللغة قسم اللغة العربية \\
\hline$r$ & المدرس أو المحاضر & المدربية والمفضل مملة الشهادة الجامعية الأولى من قسم اللغة \\
\hline$\varepsilon$ & الوسائل أو التسهيلات & 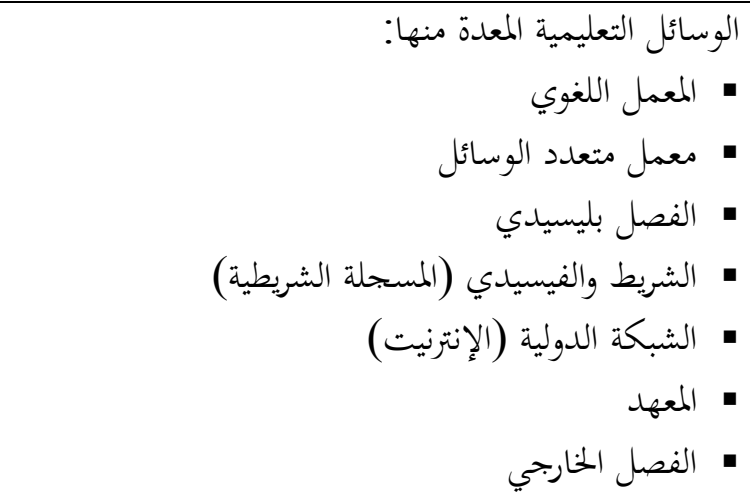 \\
\hline 0 & التكلفة & التكلفة من الحكومة بكوها الجامعة الحكومية وكذلك من الدراسية التي دفعها الطلاب في كل مستوى. \\
\hline 7 & الثقافة & 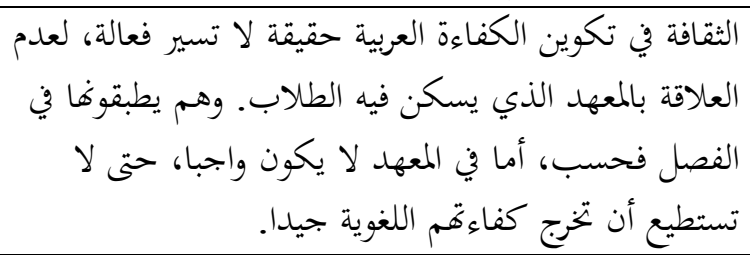 \\
\hline$V$ & الإدارة & 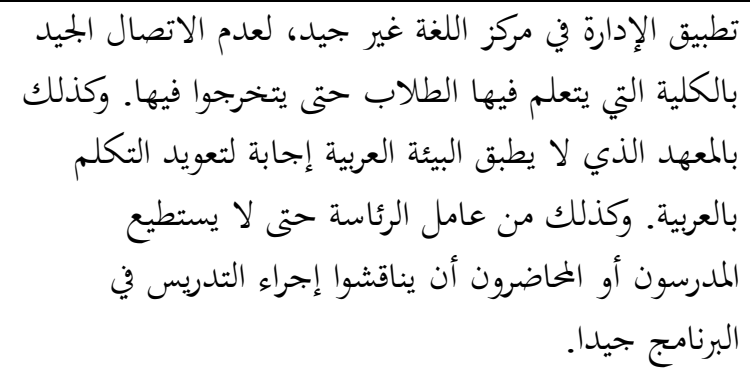 \\
\hline$\Lambda$ & عملية التدريس & التقدم المادة بنظرية الوحدات، حتى يكون تدريس المهارات \\
\hline 9 & النتيججة & 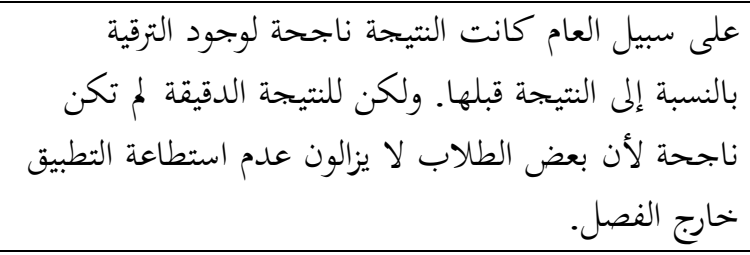 \\
\hline
\end{tabular}




\begin{tabular}{|c|c|c|}
\hline الرقم & عناصر إدارة البرنامج المكثف & العناصر في مركز اللغة قسم اللغة العربية \\
\hline 1. & الأثر & 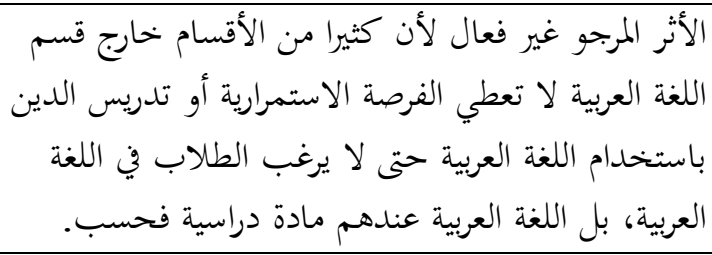 \\
\hline 11 & البيئة & 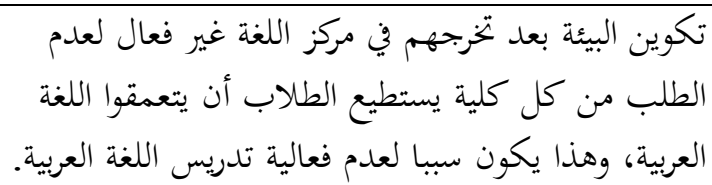 \\
\hline
\end{tabular}

○. إجراء سلسلة تنمية إدارة البرنامج المكثف العربي

\begin{tabular}{|c|c|c|}
\hline 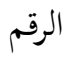 & سلسلة التنمية & سلسلة التنمية في مركز اللغة \\
\hline 1 & مرحلة التحليل & \\
\hline & • & تأسيس البرنامج تصوير التخطيط من الجحامعة الإسلامية \\
\hline & ل تحليل الواجبة & لاستيفاء الهدف السابق، فتعطي الجامعة الإسالامية اللحومية تولونج أغونج التفويض لمركز اللغة للقيام بتنفيذه. \\
\hline & • & 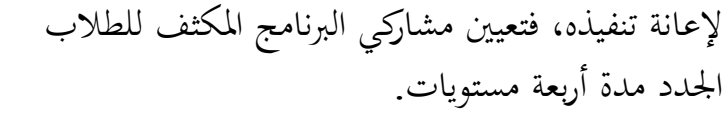 \\
\hline$r$ & مرحلة التنمية & وتركيب المقترح الذي يكتوي على تقديم برنامج مكثف عربي \\
\hline$r$ & مرحلة التصميم & داخلي مركز اللغة \\
\hline & • رمز وتعيين الهدف & قالقدرة مركز اللغة بتركيب هدف المهارات اللغوية الأربع. برجين بعد تعلمهم فيه \\
\hline & • - ت تخطيط البرنامج المكثف & 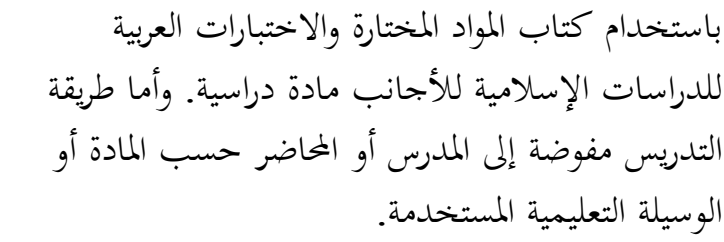 \\
\hline & " خطة البرنامج المكثف & زمن الدراسة خخطط مدة أربعة مستويات ومكان الدراسة في \\
\hline
\end{tabular}




\begin{tabular}{|c|c|c|}
\hline 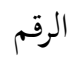 & سلسلة التنمية & سلسلة التنمية في مركز اللغة \\
\hline & العربي & الغرف الدراسية بالجامعة الإسلامية الحكومية تولونج أغونج. \\
\hline & • بلوغ الخطة الأساسية & قد بلغت الخطة الأساسية حسب التصميم المركب. \\
\hline \multirow[t]{4}{*}{$\varepsilon$} & مرحلة التطبيق & \\
\hline & • عدد اليوم واللقاء & كل كدد اليومين في الأسبوع من يوم الإثنين إلى يوم الجمعة، في \\
\hline & • عدد المواد الدراسية & والومن الوحدة الأولى إلى الوحدة الثانية عشرة للمجلد الأول، \\
\hline & • البرناميل سيناريو نشاط المكثف العربي & التقنية في الفصل الأكاديمية مباشرة اتباعا بكفاءة الطلاب، وأما \\
\hline 0 & مرحلة التقويم & بنفسه بعداء التقويم أربع مرات في السنة، ولكن إجراء التقويم النقيم \\
\hline 7 & مرحلة الإعادة والتصحيح & فعتالا، بل وجبر التدريس في البرنامج المكثف رتيب. وعدم التصحيح \\
\hline
\end{tabular}

الخلاصة

إجراء برنامج يجتاج إلى التفكير ذي مركز (اجتماعي) ويعطي المساهمة، حتى تكون تنيجة التفكير يستطيع تحقيقها في برنامج مكثف بالتركيز على الإدارة الجيدة. لإصدار البرنامج المكثف العربي الفعال فينبغي الاهتمام هذه الأشياء وهي: أولا، وجود جماعة عربية صافية خثرة. ثانيا، زمن التدريس في البرنامج المكثف، والرتيب، والمكتظّ. ثالثا، طول زمن الدراسة. رابعا، همة عالية غير منطفئة. إجراء البرنامج المكثف العربي في الجامعة الإسلامية الحكومية تولونج مازال غير جيد، بسبب وجود المشكات في أثناء إجرائه. وجود المشكلات التي توصل إلى أن يكون برنابحا جيدا، وهو التنسيق بين العناصر في تلك الجامعة بنفسها غير منفذ ويسبب هذا البرنامج يصيبه عدم الاستمرار. وظهرت المشكلة عندما كانت المواد المدروسة غير مستمرة ومعودة في المعهد الذي يسكن فيه الطلاب، وكذلك استمرار كفاءة اللغة العربية عن وسيلة تدريس الدين الإسلامي باستخدام اللغة العربية في الكلية. حتى 
تكون رغبة بعض الطلاب في اللغة العربية غير مكونة جدية بسبب أفهم يشعرون بعدم اللقاء أو

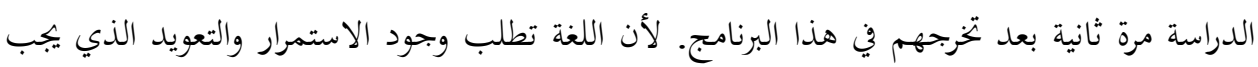
عليها المواصلة خارج مساحتها أو منطقتها. وبذلك تشدد إدارة البرنامج المكثف العربي على استيفاء حوائج الطلاب، وإجراءها بإدارة

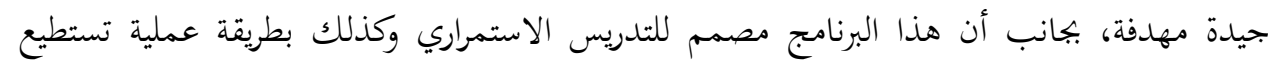

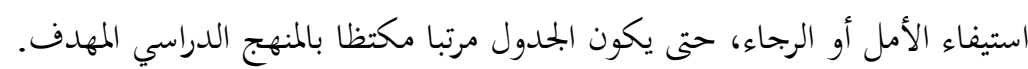

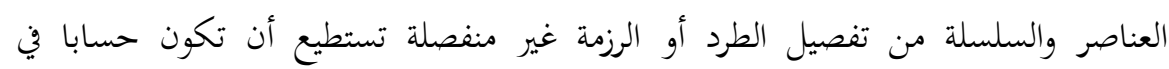

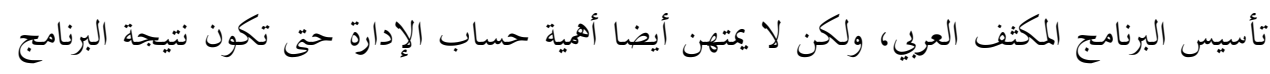
صائبة ومهدفة.

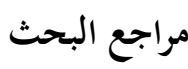

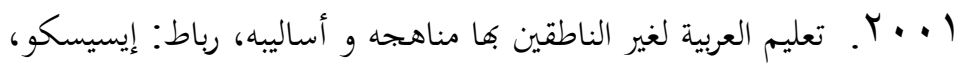

منشورات المنظمة الإسلامية للتربية والعلوم والثقافة.

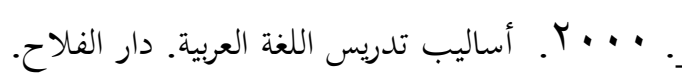

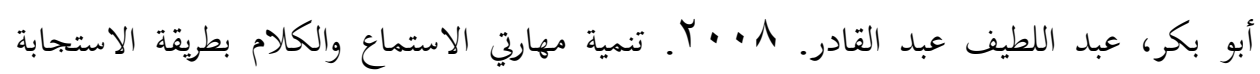

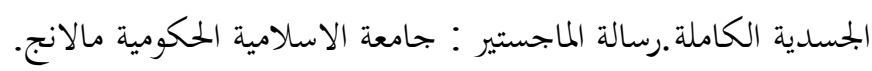

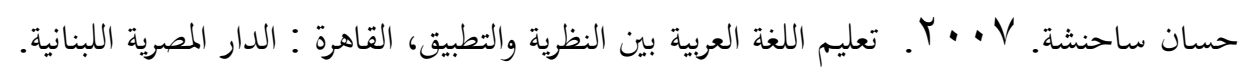

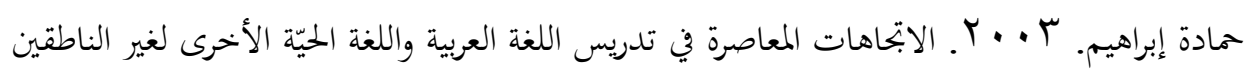
بها. القاهرة : دار الفكر العربي.

الخولى، محمد على. . . . ب. الاختبارات اللغوية، (دون المدينة: دار الفلاح للنشر والتوزيع.

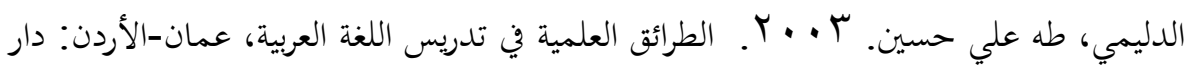
الشروق.

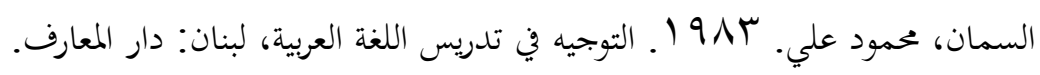
شحاتة، حسن. Y · · Y. تعليم اللغة العربية بين النظرية والتطبيق. القاهرة: الدارالمصرية اللبنانية.

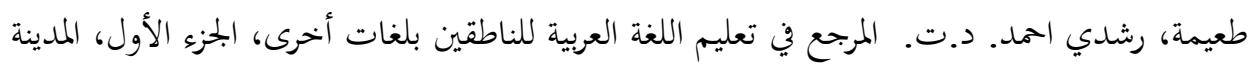
المنورة: جامعة أم القرى معهد اللغة العربية.

عبد العزيز بن إبراهيم العصلي. Y + . Y. طرائق تدريس اللغة العربية للناطقين بلغات أخرى، رياض: 


$$
\text { جامعة الإمام محمد بن سعود الاسلامي. }
$$

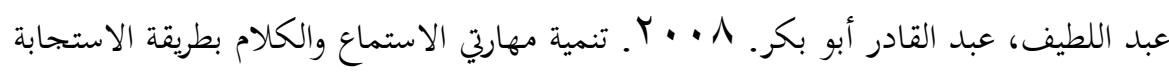

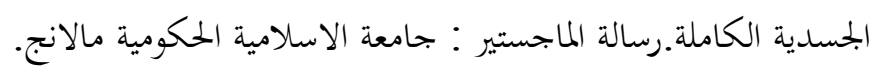

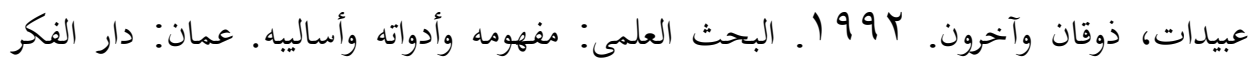
للنشر والتوزيع.

$$
\begin{aligned}
& \text { عليان، أحمد فؤاد. بو } 99 \text { 1. المهارات اللغوية ماهيتها و طرائق تدريسها، الرياض: دار المسلم. }
\end{aligned}
$$

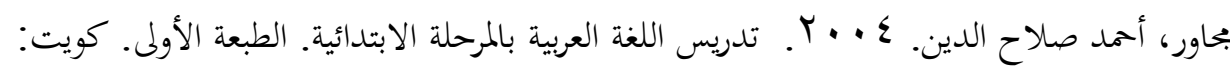

$$
\begin{aligned}
& \text { دار القلم. } \\
& \text { محمود كامل الناقة. .... }
\end{aligned}
$$

لعربية للناطقين بلغات أخرى، أسسه ومداخله وطرق تدريسه، مكة المكرمة : جامعة أم القرى.

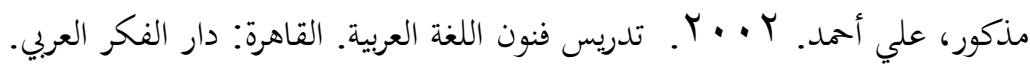

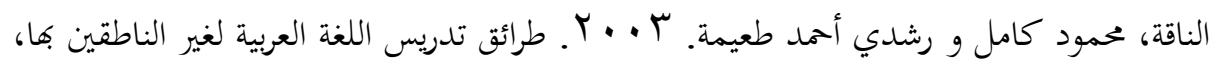
مصر: ايسيسكو.

الناقة، محمود كامل. 910 19. تعليم اللغة العربية للناطقين بلغة أخرى. مكة المكرمة: جامعة أم القرى. يونس، فتحي على ومحمود كامل الناقة. 0 ○ · Y. أساسيات تعليم اللغة العربية، القاهرة : دار الثقافة.

Borg, Walter R., and Gall, M. D., (1983). Educational Researcb: an Introduction $\left(4^{e d}\right)$, New York and London: Longman.

Depag RI., (2007). Al-Quran dan Terjemahnya dengan Transliterasi Arab-Latin, Bandung: Gema Risalah Press.

Faisal, Sanapiah., (2003). Metodologi Penelitian Pendidikan, Balai Pustaka, Jakarta.

Gay, L. R., (1991). Educational Evaluation and Measurement: Competencies for Analysis and Application. Second Edition. Ney York: Macmillan.

Tim Puslitjaknov., (2008). Metode Penelitian Pengembangan, Jakarta: Balitbang Depdiknas. 